\title{
Stability of critical behaviour of weakly disordered systems with respect to the replica symmetry breaking
}

\author{
V.V. Prudnikov, P.V. Prudnikov, and A.A. Fedorenko \\ Department of Theoretical Physics, Omsk State University, Mira prospekt 55-A, Omsk, 644077, \\ Russia
}

(January 25, 2001)

\begin{abstract}
A field-theoretic description of the critical behaviour of the weakly disordered systems is given. Directly, for three- and two-dimensional systems a renormalization analysis of the effective Hamiltonian of model with replica symmetry breaking (RSB) potentials is carried out in the two-loop approximation. For case with 1-step RSB the fixed points (FP's) corresponding to stability of the various types of critical behaviour are identified with the use of the Pade-Borel summation technique. Analysis of FP's has shown a stability of the critical behaviour of the weakly disordered systems with respect to RSB effects and realization of former scenario of disorder influence on critical behaviour.
\end{abstract}

64.60.Ak, 64.60.-i, 64.60.Fr, 64.60.Ht

Typeset using REVTEX 
The effects produced by weak quenched disorder on the critical phenomena have been studied since many years ago [1 [5]. According to the Harris criterion [1], the disorder effects the critical behaviour only if $\alpha$, the specific heat exponent of the pure system, is positive. In this case a new universal critical behaviour, with new critical exponents, was established. In contrast, when $\alpha<0$, the disorder appears to be irrelevant for the critical behaviour.

In dealing with the weak quenched disorder the traditional approach is the replica method [4. [5], and in terms of replicas all the results obtained for the disorder systems correspond to the so-called replica-symmetric (RS) solutions. Physically it means that only unique ground state is assumed to be relevant for the observable thermodynamics. However, in a number of papers [6 8] the ideas about replica symmetry breaking (RSB) in the systems with quenched disorder were presented. For the first time in the paper [6] physical arguments showing that in the presence of the quenched disorder there exist numerous local minimal energy configurations separated by finite barriers and demonstrating how the summation over these local minima configurations can provide additional RSB interaction potentials for fluctuating fields have been offered. The renormalization group (RG) description of the classical $\phi^{4}$ model with RSB potentials was presented in the one-loop approximation using $\varepsilon$ - expansion [6] [8]. It was shown that the RSB degrees of freedom produce dramatic effect on the asymptotic behaviour of the RG flows, such that for a general type of the RSB there exist no stable fixed points, and RG equations arrive into the strong coupling regime. In contrast, in Ref. [9] using of the fermion representation it was demonstraited that the critical behaviour of the 2D weakly disordered Ising system is stable with respect to the RSB modes.

However, our numerous investigations of pure and disordered systems performed in the two-loop and higher orders of the approximation for the 3D system directly together with methods of series summation show that the predictions made in the lowest order of the approximation, especially on the basis of the $\varepsilon$ - expansion, can differ strongly from the real critical behaviour [10]. Therefore, the results of RSB effects investigation in Refs. [6 8] must be reconsidered with the use of a more accurate field-theory approach in the higher orders of the approximation.

In this paper, we have realized the field-theoretical RG description in the two-loop approximation of the 3D and 2D models of the weakly disordered systems with RSB interaction potentials of forth order on fluctuating fields. We have carried out the solution of the RG equations with the use of series summation method and the analysis of stability of various types of critical behaviour with respect to RSB effects.

We consider an $O(p)$ - symmetric Ginzburg-Landau-Wilson model of a spin system with weak quenched disorder near critical point given by the Hamiltonian

$$
H=\int d^{d} x\left\{\frac{1}{2} \sum_{i=1}^{p}\left[\nabla \phi_{i}(x)\right]^{2}+\frac{1}{2}[\tau-\delta \tau(x)] \sum_{i=1}^{p} \phi_{i}^{2}(x)+\frac{1}{4} g \sum_{i, j=1}^{p} \phi_{i}^{2}(x) \phi_{j}^{2}(x)\right\},
$$

where $\phi_{i}(x)$ is the $p$-component order parameter and $\delta \tau(x)$ is the Gaussian-distributed random transition temperature with the second moment of distribution $\left\langle\left\langle(\delta \tau(x))^{2}\right\rangle\right\rangle \sim u$ defined by the positive constant $u$ which is proportional to the concentration of defects. The use of the standard replica trick gives the possibility to average easily over the disorder and reduce the task of statistical description of the weakly disordered system with the Hamiltonian (1) to the homogeneous system with the effective Hamiltonian 


$$
H_{n}=\int d^{d} x\left\{\frac{1}{2} \sum_{i=1}^{p} \sum_{a=1}^{n}\left[\left[\nabla \phi_{i}^{a}(x)\right]^{2}+\tau\left[\phi_{i}^{a}(x)\right]^{2}\right]+\frac{1}{4} \sum_{i, j=1}^{p} \sum_{a, b=1}^{n} g_{a b}\left[\phi_{i}^{a}(x)\right]^{2}\left[\phi_{j}^{b}(x)\right]^{2}\right\},
$$

which is a functional of $n$ replications of the original order parameter with an additional vertex $u$ in the replica symmetric matrix $g_{a b}=g \delta_{a b}-u$. The properties of the original disordered system are obtained in the replica number limit $n \rightarrow 0$. The following standard RG procedure based on the statistical calculation of contribution to the partition function of longwavelength order parameter fluctuations around the global minimum state with $\phi(x)=0$ gives the possibility to find the various types of critical behaviour and conditions of their stability and carry out the calculation of critical exponents.

However, as it was shown in Refs. [6 8], the fluctuations of random transition temperature $\delta \tau(x)$ for $[\tau-\delta \tau(x)]<0$ can lead to realization in system of numerous regions with $\phi(x) \neq 0$ displaying through the numerous local minimal energy configurations separated from the ground state by finite barriers. In this case the direct application of the traditional replicasymmetric RG scheme may be questioned. For statistical description of such systems near phase transition point the Parisi RSB scheme was used in Refs. [6] 8] by analogy with spin glasses [11]. It was argued that spontaneous RSB can occur due to the interaction of the fluctuating fields with the local non-perturbative degrees of freedom coming from the multiple local minima solutions of the mean-field equations. It was shown that the summation over these solutions in the replica partition function can provide the additional non-trivial RSB potential $\sum_{a, b} g_{a b} \phi_{a}^{2} \phi_{b}^{2}$ in which the matrix $g_{a b}$ has the Parisi RSB structure [11]. According to the technique of the Parisi RSB algebra, in the limit $n \rightarrow 0$ the matrix $g_{a b}$ is parametrized in terms of its diagonal elements $\tilde{g}$ and the off-diagonal function $g(x)$ defined in the interval $0<x<1: g_{a b} \rightarrow(\tilde{g}, g(x))$. The operations with the matrices $g_{a b}$ are given by the following rules:

$$
g_{a b}^{k} \rightarrow\left(\tilde{g}^{k} ; g^{k}(x)\right),\left(\hat{g}^{2}\right)_{a b}=\sum_{c=1}^{n} g_{a c} g_{c b} \rightarrow(\tilde{c} ; c(x)),\left(\hat{g}^{3}\right)_{a b}=\sum_{c, d=1}^{n} g_{a c} g_{c d} g_{d b} \rightarrow(\tilde{d} ; d(x)),
$$

where

$$
\begin{array}{r}
\tilde{c}=\tilde{g}^{2}-\int_{0}^{1} d x g^{2}(x), \quad c(x)=2\left[\tilde{g}-\int_{0}^{1} d y g(y)\right] g(x)-\int_{0}^{x} d y[g(x)-g(y)]^{2}, \\
\tilde{d}=\tilde{c} \tilde{g}-\int_{0}^{1} d x c(x) g(x), \quad d(x)=\left[\tilde{g}-\int_{0}^{1} d y g(y)\right] c(x)+\left[\tilde{c}-\int_{0}^{1} d y c(y)\right] g(x) \\
-\int_{0}^{x} d y[g(x)-g(y)][c(x)-c(y)] .
\end{array}
$$

The RS situation corresponds to the case $g(x)=$ const independent of $x$.

We carried out the field-theoretical RG description of the 3D and $2 \mathrm{D}$ models with the effective replicated Hamiltonian (2) in which the matrix $g_{a b}$ has the RSB structure in the two-loop approximation. In the field-theoretic approach the asymptotic critical behavior of systems in the fluctuation region are determined by the Callan-Symanzik RG equation for the vertex parts of the irreducible Green's functions. To calculate the $\beta$ functions as functions of the renormalized elements of the matrix $g_{a b}$ appearing in the RG equation, 
we used the method based on the Feynmann diagram technique and the renormalization procedure [12]. We obtained the next expressions for two-pont vertex function $\Gamma^{(2)}$ and four-point vertex functions $\Gamma_{a b}^{(4)}$ :

$$
\begin{aligned}
& \left.\frac{\partial \Gamma^{(2)}}{\partial k^{2}}\right|_{k^{2}=0}=1+4 f g_{a a}^{2}+2 p f \sum_{c=1}^{n} g_{a c} g_{c a}, \\
& \left.\Gamma_{a b}^{(4)}\right|_{k_{i}=0}=g_{a b}-p \sum_{c=1}^{n} g_{a c} g_{c b}-4 g_{a a} g_{a b}-4 g_{a b}^{2}+(8+16 h) g_{a b}^{3}+(24+8 h) g_{a a}^{2} g_{a b} \\
& \quad+48 h g_{a a} g_{a b}^{2}+4 g_{a a} g_{b b} g_{a b}+8 p h \sum_{c=1}^{n} g_{a c} g_{c b}^{2}+8 p h g_{a b} \sum_{c=1}^{n} g_{a c} g_{c b} \\
& \quad+4 p h g_{a b} \sum_{c=1}^{n} g_{a c}^{2}+2 p \sum_{c=1}^{n} g_{a c} g_{c c} g_{c b}+4 p g_{a a} \sum_{c=1}^{n} g_{a c} g_{c b}+p^{2} \sum_{c, d=1}^{n} g_{a c} g_{c d} g_{d b},
\end{aligned}
$$

where

$$
\begin{aligned}
& f(d)=-\left.\frac{1}{J^{2}} \frac{\partial}{\partial k^{2}} \int \frac{d^{d} k_{1} d^{d} k_{2}}{\left(k_{1}^{2}+1\right)\left(k_{2}^{2}+1\right)\left(\left(k_{1}+k_{2}+k\right)^{2}+1\right)}\right|_{k^{2}=0}, \\
& h(d)=\frac{1}{J^{2}} \int \frac{d^{d} k_{1} d^{d} k_{2}}{\left(k_{1}^{2}+1\right)\left(k_{2}^{2}+1\right)\left(\left(k_{1}+k_{2}\right)^{2}+1\right)}, \\
& J=\int d^{d} k /\left(k^{2}+1\right)^{2}, \quad f(d=3)=\frac{2}{27}, \quad h(d=3)=\frac{2}{3}, \\
& f(d=2)=0.11464, \quad h(d=2)=0.78129,
\end{aligned}
$$

and was made the redefinition $g_{a b} \rightarrow g_{a b} / J$.

However, the renormalization procedure for vertex functions is made difficult because of complicated expressions (3)-(5) for the operations with the matrices $g_{a b}$. The step-like structure of the function $g(x)$ revealed in Refs. [6 8 gives the possibility to realize the renormalization procedure. In this paper we considered only the matrices $g_{a b}$ which have the structure known as the 1 step RSB with function $g(x)$ of the next view:

$$
g(x)= \begin{cases}g_{0}, & 0 \leq x<x_{0} \\ g_{1}, & x_{0}<x \leq 1\end{cases}
$$

where $0 \leq x_{0} \leq 1$ is the coordinate of the step and it remains arbitrary parameter. The value of $x_{0}$ does not be changed during the renormalization procedure and remains the same as in the starting function $g_{0}(x)$. In consequence the $\mathrm{RG}$ transformations of the effective replicated Hamiltonian with RSB potentials are determined by the three parameters $\tilde{g}, g_{0}$ and $g_{1}$.

The critical properties of model can be extracted from the coefficients $\beta_{i}\left(\tilde{g}, g_{0}, g_{1}\right)(i=$ $1,2,3), \gamma_{\phi}\left(\tilde{g}, g_{0}, g_{1}\right)$, and $\gamma_{\phi^{2}}\left(\tilde{g}, g_{0}, g_{1}\right)$ of the Callan-Symanzik RG equation. We obtained the $\beta$ functions in the two-loop approximation in the form of the expansion series in renormalized parameters $\tilde{g}, g_{0}$ and $g_{1}$

$$
\begin{aligned}
\beta_{1} & =-\tilde{g}+(8+p) \tilde{g}^{2}-p x_{0} g_{0}{ }^{2}-p\left(1-x_{0}\right) g_{1}{ }^{2}+((8 f-40 h+20) p \\
& +16 f-176 h+88) \tilde{g}^{3}+(24 h-8 f-12) x_{0} p \tilde{g} g_{0}^{2}+(24 h-8 f
\end{aligned}
$$




$$
\begin{aligned}
& -12)\left(1-x_{0}\right) p \tilde{g} g_{1}^{2}+(16 h-8) x_{0} p g_{0}{ }^{3}+(16 h-8)\left(1-x_{0}\right) p g_{1}{ }^{3}, \\
\beta_{2} & =-g_{0}+(4+2 p) \tilde{g} g_{0}-\left(2 p x_{0}-4\right) g_{0}{ }^{2}-2\left(1-x_{0}\right) p g_{0} g_{1} \\
& +((8 f-48 h+28) p+16 f-48 h+24) \tilde{g}^{2} g_{0}+\left(\left((32 h-16) x_{0}\right.\right. \\
& +8-32 h) p+48-96 h) \tilde{g} g_{0}^{2}+(32 h-16)\left(1-x_{0}\right) p \tilde{g} g_{0} g_{1} \\
& +\left((48 h-8 f-20) x_{0} p-32 h+16\right) g_{0}{ }^{3}+(32 h-8)\left(1-x_{0}\right) p g_{0}{ }^{2} g_{1} \\
& +(16 h-12-8 f)\left(1-x_{0}\right) p g_{0} g_{1}^{2}, \\
\beta_{3} & =-g_{1}-p x_{0} g_{0}^{2}+\left(p\left(x_{0}-2\right)+4\right) g_{1}{ }^{2}+(4+2 p) \tilde{g} g_{1}+((8 f-48 h \\
& +28) p+16 f-48 h+24) g_{1} \tilde{g}^{2}+(16 h-8) x_{0} p \tilde{g} g_{0}{ }^{2}+\left(\left((8-16 h) x_{0}\right.\right. \\
& -8) p+48-96 h) u_{0} g_{1}{ }^{2}+(16 h-8) x_{0} p g_{0}{ }^{3}+(8 h-8 f-4) x_{0} p g_{1} g_{0}{ }^{2} \\
& +\left((8 f-24 h+12) x_{0} p+(48 h-8 f-20) p+16-32 h\right) g_{1}{ }^{3} .
\end{aligned}
$$

By analogy with papers [6-8] we changed $g_{a \neq b} \rightarrow-g_{a \neq b}$ in the expressions (10) for the $\beta$ functions, so that the off-diagonal elements $g_{a \neq b}$ would be positively defined.

It is well known that perturbation series are asymptotic series, and that the vertices describing the interaction of the order parameter fluctuations in the fluctuating region $\tau \rightarrow 0$ are large enough so that expressions (10) cannot be used directly. For this reason, to extract the required physical information from the obtained expressions, we employed the PadéBorel approximation of the summation of asymptotic series extended to the multiparameter case. The direct and inverse Borel transformations for the multiparameter case have the form

$$
\begin{aligned}
& f\left(\tilde{g}, g_{0}, g_{1}\right)=\sum_{i, j, k} c_{i j k} \tilde{g}^{i} g_{0}^{j} g_{1}^{k}=\int_{0}^{\infty} e^{-t} F\left(\tilde{g} t, g_{0} t, g_{1} t\right) d t \\
& F\left(\tilde{g}, g_{0}, g_{1}\right)=\sum_{i, j, k} \frac{c_{i j k}}{(i+j+k) !} \tilde{g}^{i} g_{0}^{j} g_{1}^{k}
\end{aligned}
$$

A series in the auxiliary variable $\theta$ is introduced for analytical continuation of the Borel transform of the function:

$$
\tilde{F}\left(\tilde{g}, g_{0}, g_{1}, \theta\right)=\sum_{k=0}^{\infty} \theta^{k} \sum_{i=0}^{k} \sum_{j=0}^{k-i} \frac{c_{i, j, k-i-j}}{k !} \tilde{g}^{i} g_{0}^{j} g_{1}^{k-i-j}
$$

to which the $[\mathrm{L} / \mathrm{M}]$ Padé approximation is applied at the point $\theta=1$. To perform the analytical continuation, the Padé approximant of [L/1] type may be used which is known to provide rather good results for various Landau-Wilson models (see, e.g. Refs. [13, 14]). The property of preserving the symmetry of a system during application of the Padé approximation by the $\theta$ method, as in [13], has become important for multivertices models. We used the $[2 / 1]$ approximant to calculate the $\beta$ functions in the two-loop approximation.

The nature of the critical behavior is determined by the existence of a stable FP satisfying the system of equations

$$
\beta_{i}\left(\tilde{g}^{*}, g_{0}^{*}, g_{1}^{*}\right)=0 \quad(i=1,2,3)
$$

for resummed $\beta$ functions. We have found three types of non-trivial FP's in the physical region of parameter space $\tilde{g}^{*}, g_{0}^{*}, g_{1}^{*} \geq 0$ for different values of $p=1,2,3$, which are presented 
in Tables $\mathbb{4} \mathbb{W}$ (the exception was made in the case with $p=3$, when presented in the Table III the coordinates of type II and type III FP's are characterized by unphysical negative values of $g_{0}^{*}$ and $\left.g_{1}^{*}\right)$. Type I with $\tilde{g}^{*} \neq 0, g_{0}^{*}=g_{1}^{*}=0$ corresponds to the RS FP of a pure system, type II with $\tilde{g}^{*} \neq 0, g_{0}^{*}=g_{1}^{*} \neq 0$ is a disorder-induced RS FP, and type III with $\tilde{g}^{*} \neq 0, g_{0}^{*}=0, g_{1}^{*} \neq 0$ corresponds to the 1 -step RSB FP. The values of parameters $\tilde{g}^{*}, g_{1}^{*}$ for the 1-step RSB FP depend on the coordinate of the step $x_{0}$, and we presented in Tables $\mathbb{Q} \mathbb{\square}$ the received values of these parameters in the range $0 \leq x_{0} \leq 1$ with changes through the step $\Delta x_{0}=0.1$.

The type of critical behavior of this disordered system for each value of $p$ is determined by the stability of the corresponding FP. The requirement that the FP be stable reduces to the condition that the eigenvalues $\lambda_{i}$ of the matrix

$$
B_{i, j}=\frac{\partial \beta_{i}\left(\tilde{g}^{*}, g_{0}^{*}, g_{1}^{*}\right)}{\partial g_{j}}
$$

lie in the right-hand side complex half-plane. Analysis of the received values $\lambda_{i}$ for FP's in Tables $\mathbb{E V}$ shows that for 3D and 2D Ising models $(p=1)$ and 3D XY model $(p=2)$ the disorder-induced RS FP's are stable. However, we believe that in the higher field-theory orders of approximation the RS FP of a pure system will be stable for the 3D XY model. Two facts indicate this, such as the weak stability of the disorder-induced RS FP and that in the two-loop approximation the marginal value of $p_{c}=2.0114$ for the borderline between regions of stability for the disorder-induced RS FP and the RS FP of a pure system. In the higher orders of approximation the marginal value of $p_{c}<2$ such as the specific heat exponent $\alpha>0$ for the pure XY model. For the 3D Heisenberg model $(p=3)$ the RS FP of a pure system is stable and both another types of FP's are characterized by unphysical values of coordinates $g_{0}^{*}$ and $g_{1}^{*}$. Our conclusions coincide with results of Ref. [9] for the 2D random Ising model, but contradict with results of Refs. [6 8] for 3D disordered systems.

However, we must note that the obtained RS FP values for vertices $\tilde{g}, g_{0}$ and the eigenvalues $\lambda_{1}$ and $\lambda_{2}$ of the stability matrix correspond to results of Ref. [15], in which a fieldtheoretic treatment of disordered 3D and 2D spin systems was presented in the two-loop approximation. The vertices $v_{1}$ and $v_{2}$ introduced in Ref. [15]] are connected with the vertices $\tilde{g}$ and $g_{0}$ by the relations $v_{1}=(p+8)\left(\tilde{g}+g_{0}\right)$ and $v_{2}=8 g_{0}$. We have calculated the static critical exponents from the resummed by the generalized Padé-Borel method $\gamma$ functions in the corresponding stable RS FP's (Table VD. For comparison we presented also in Table $\square$ a values of the critical exponents from [16.17 received for pure and disordered 3D systems without RSB in the six-loop approximation. Comparison of the exponent values shows that their differences are not more than 0.01. It gives the possibility to consider our results of the RSB effects investigation as reliable. The model with RSB potentials is the another one example of the multivertices models [13] for which the predictions made on the basis of the $\varepsilon$ - expansion can differ strongly from results of the use a more accurate field-theory approach for the 3D system directly together with methods of series summation. This situation is explained by a competition of numerous types of FP's in the parameter space of the multivertices models. Therefore, the spread of results of the $\varepsilon$ - expansion from $\varepsilon \ll 1$ to $\varepsilon=1$ is impossible, as a rule, without intersection of the stability ranges for the various types of FP's.

Thus, the RG investigations carried out in the two-loop approximation show the stability of the critical behaviour of weakly disordered systems with respect to the RSB effects. 
In dilute Ising-like systems the disorder-induced critical behaviour is realized with RS FP. The weak disorder is irrelevant for the critical behaviour of systems with multicomponent order parameter although the proof of it for 3D systems with two-component order parameter demands a calculations in the higher orders of approximation. The possible influence the RSB degrees of freedom on the critical behaviour of highly disordered systems can be nonperturbatively revealed by the use of the Monte Carlo simulation method [18 for definition of the probability distributions for order parameter and random transition temperature fluctuations.

\section{ACKNOWLEDGMENTS}

We would like to thank the Russian Foundation for Basic Research for support through Grant No. 00-02-16455. 


\section{REFERENCES}

[1] A. B. Harris, J. Phys. C 7, 1671 (1974).

[2] A. B. Harris and T. C. Lubensky, Phys.Rev.Lett. 33, 1540 (1974).

[3] D. E. Khmelnitskii, Sov. Phys. JETP 41981 (1975).

[4] J. Emery, Phys. Rev. B 11, 239 (1975).

[5] G. Grinstein and A. Luther, Phys. Rev. B 13, 1329 (1976).

[6] Vik. S. Dotsenko, A. B. Harris, D. Sherrington, and R. B. Stinchcombe, J. Phys. A 28, 3093 (1995).

[7] Vik. S. Dotsenko and D. E. Feldman, J. Phys. A 28, 5183 (1995).

[8] Vik. S. Dotsenko, Uspekhi Fizicheskikh Nauk 165, 481 (1995).

[9] D. E. Feldman, A. V. Izyumov, and Vik. S. Dotsenko, e-print cond-mat/9512158.

[10] V. V. Prudnikov, A. V. Ivanov, and A. A. Fedorenko, Sov. Phys. JETP Lett. 66835 (1997); V. V. Prudnikov, S. V. Belim, A. V. Ivanov, E. V. Osintsev, A. A. Fedorenko, Sov. Phys. JETP 87527 (1998); V. V. Prudnikov, P. V. Prudnikov, and A. A. Fedorenko, Sov. Phys. JETP Lett. 68950 (1998); Phys.Rev. B 62, 8777 (2000).

[11] M. Mezard, G. Parisi, and M. Virasoro, Spin-Glass Theory and Beyond (Singapore, World Scientific, 1987); M. Mezard and G. Parisi, J.Phys. I (France) 1, 809 (1991); M. Mezard and A.P.Young, Europhys.Lett. 18, 653 (1992); Vik. S. Dotsenko, Uspekhi Fizicheskikh Nauk 163, 1 (1993); Vik. S. Dotsenko, Introduction to the Theory of SpinGlasses and Neural Networks (Singapore, World Scientific, 1994).

[12] J. Zinn-Justin, Quantum field theory and critical phenomena (Clarendon Press, Oxford, 1996).

[13] S. A. Antonenko and A. I. Sokolov, Phys.Rev. B 49, 15901 (1994); A.I. Sokolov, K.B. Varnashev, A.I. Mudrov. Int. J. Mod. Phys. B 12, 1365 (1998); A.I. Sokolov, K.B. Varnashev, Phys.Rev. B 59, 8363 (1999).

[14] G. A. Baker, B. G. Nickel, and D. I. Meiron, Phys. Rev. B 17, 1365 (1978).

[15] G. Jug, Phys. Rev. B 27, 609 (1983).

[16] J. C. LeGuillou and J. Zinn-Justin, Phys. Rev. B 21, 3976 (1980).

[17] A. Pelissetto and E. Vicari, e-print cond-mat/0002402.

[18] M.M. Tsypin, Phys. Rev. B 55, 8911 (1997). 


\section{TABLES}

TABLE I. Coordinates of the FP's and eigenvalues of the stability matrix for the 3D Ising model.

\begin{tabular}{|c|c|c|c|c|c|c|}
\hline Type & $x_{0}$ & $\tilde{g}^{*}$ & $g_{0}^{*}$ & $g_{1}^{*}$ & $\lambda_{2}$ & $\lambda_{3}$ \\
\hline 1 & & 0.1774103 & 0 & 0 & -0.16924 & -0.16924 \\
\hline 2 & & 0.1843726 & 0.0812240 & 0.0812240 & $0.525 \pm 0.089 i$ & 0.211 \\
\hline \multirow[t]{11}{*}{3} & 0.0 & 0.1843726 & 0 & 0.0812240 & $0.525319 \pm 0.089273 i$ & -0.039167 \\
\hline & 0.1 & 0.1839722 & 0 & 0.0829404 & $0.535185 \pm 0.098291 i$ & -0.049185 \\
\hline & 0.2 & 0.1835134 & 0 & 0.0846432 & $0.547065 \pm 0.106665 i$ & -0.059851 \\
\hline & 0.3 & 0.1829917 & 0 & 0.0863186 & $0.560666 \pm 0.113305 i$ & -0.071187 \\
\hline & 0.4 & 0.1824035 & 0 & 0.0879503 & $0.576473 \pm 0.118038 i$ & -0.083210 \\
\hline & 0.5 & 0.1817458 & 0 & 0.0895200 & $0.595060 \pm 0.120271 i$ & -0.095927 \\
\hline & 0.6 & 0.1810165 & 0 & 0.0910067 & $0.617241 \pm 0.118872 i$ & -0.109334 \\
\hline & 0.7 & 0.1802154 & 0 & 0.0923872 & $0.643936 \pm 0.111389 i$ & -0.123415 \\
\hline & 0.8 & 0.1793442 & 0 & 0.0936384 & $0.675972 \pm 0.092079 i$ & -0.138133 \\
\hline & 0.9 & 0.1784070 & 0 & 0.0947426 & $0.713456 \pm 0.035266 i$ & -0.153431 \\
\hline & 1.0 & 0.1774103 & 0 & 0.0956920 & $0.857325 \quad 0.65355$ & -0.169237 \\
\hline
\end{tabular}

TABLE II. Coordinates of the FP's and eigenvalues of the stability matrix for the 3D XY model.

\begin{tabular}{cllcccrr}
\hline \hline Type & $x_{0}$ & $\tilde{g}^{*}$ & $g_{0}^{*}$ & $g_{1}^{*}$ & $\lambda_{1}$ & \multicolumn{1}{c}{$\lambda_{2}$} & $\lambda_{3}$ \\
\hline 1 & & 0.1558303 & 0 & 0 & 0.667315 & -0.001672 & -0.001672 \\
2 & & 0.1558310 & 0.0005837 & 0.0005837 & 0.667312 & 0.001682 & 0.000004 \\
3 & 0.0 & 0.1558310 & 0 & 0.0005837 & 0.667313 & 0.001683 & -0.000001 \\
& 0.1 & 0.1558310 & 0 & 0.0006143 & 0.667313 & 0.001684 & -0.000088 \\
& 0.2 & 0.1558310 & 0 & 0.0006483 & 0.667313 & 0.001685 & -0.000186 \\
& 0.3 & 0.1558310 & 0 & 0.0006863 & 0.667313 & 0.001686 & -0.000296 \\
& 0.4 & 0.1558310 & 0 & 0.0007291 & 0.667313 & 0.001687 & -0.000419 \\
& 0.5 & 0.1558310 & 0 & 0.0007775 & 0.667313 & 0.001687 & -0.000559 \\
& 0.6 & 0.1558309 & 0 & 0.0008327 & 0.667313 & 0.001688 & -0.000717 \\
& 0.7 & 0.1558308 & 0 & 0.0008964 & 0.667314 & 0.001690 & -0.000901 \\
& 0.8 & 0.1558307 & 0 & 0.0009707 & 0.667314 & 0.001692 & -0.001116 \\
& 0.9 & 0.1558306 & 0 & 0.0010583 & 0.667315 & 0.001694 & -0.001369 \\
& 1.0 & 0.1558303 & 0 & 0.0011633 & 0.667316 & 0.001696 & -0.001672 \\
\hline \hline
\end{tabular}

TABLE III. Coordinates of the FP's and eigenvalues of the stability matrix for the 3D Heisenberg model.

\begin{tabular}{cllcccrr}
\hline \hline Type & $x_{0}$ & $\tilde{g}^{*}$ & $g_{0}^{*}$ & $g_{1}^{*}$ & $\lambda_{1}$ & $\lambda_{2}$ & \multicolumn{1}{c}{$\lambda_{3}$} \\
\hline 1 & & 0.1382700 & 0 & 0 & 0.681378 & 0.131537 & 0.131537 \\
2 & & 0.1419323 & -0.0358629 & -0.0358629 & 0.672676 & -0.089135 & -0.005783 \\
3 & 0.0 & 0.1419323 & 0 & -0.0358629 & 0.672676 & -0.089135 & -0.005783 \\
& 0.1 & 0.1419931 & 0 & -0.0381865 & 0.672729 & -0.086515 & 0.001104 \\
& 0.2 & 0.1420386 & 0 & -0.0408334 & 0.672845 & -0.083560 & 0.008802 \\
& 0.3 & 0.1420600 & 0 & -0.0438761 & 0.673046 & -0.080206 & 0.017469 \\
& 0.4 & 0.1420441 & 0 & -0.0474104 & 0.673361 & -0.076366 & 0.027299 \\
& 0.5 & 0.1419699 & 0 & -0.0515650 & 0.673831 & -0.071931 & 0.038540 \\
& 0.6 & 0.1418040 & 0 & -0.0565177 & 0.674509 & -0.066754 & 0.051518 \\
& 0.7 & 0.1414913 & 0 & -0.0625193 & 0.675476 & -0.060636 & 0.066656 \\
& 0.8 & 0.1409374 & 0 & -0.0699349 & 0.676837 & -0.053299 & 0.084517 \\
& 0.9 & 0.1399720 & 0 & -0.0793131 & 0.678742 & -0.044336 & 0.105835 \\
& 1.0 & 0.1382700 & 0 & -0.0915089 & 0.681378 & -0.033124 & 0.131537 \\
\hline \hline
\end{tabular}


TABLE IV. Coordinates of the FP's and eigenvalues of the stability matrix for the 2D Ising model.

\begin{tabular}{cllcccrr}
\hline \hline Type & $x_{0}$ & $\tilde{g}^{*}$ & $g_{0}^{*}$ & $g_{1}^{*}$ & $\lambda_{1}$ & $\lambda_{2}$ & $\lambda_{3}$ \\
\hline 1 & & 0.2693889 & 0 & 0 & 0.461180 & -0.095180 & -0.095180 \\
\hline 2 & & 0.2735768 & -0.0645918 & -0.0645918 & 0.446348 & 0.162092 & 0.056959 \\
\hline 3 & 0.0 & 0.2735768 & 0 & -0.0645918 & 0.446348 & 0.162092 & -0.025112 \\
& 0.1 & 0.2733970 & 0 & -0.0665073 & 0.446636 & 0.165301 & -0.029995 \\
& 0.2 & 0.2731825 & 0 & -0.0685156 & 0.447048 & 0.168803 & -0.035240 \\
& 0.3 & 0.2729279 & 0 & -0.0706204 & 0.447615 & 0.172637 & -0.040881 \\
& 0.4 & 0.2726271 & 0 & -0.0728248 & 0.448377 & 0.176842 & -0.046958 \\
& 0.5 & 0.2722731 & 0 & -0.0751310 & 0.449385 & 0.181468 & -0.053513 \\
& 0.6 & 0.2718579 & 0 & -0.0775405 & 0.450704 & 0.186566 & -0.060592 \\
& 0.7 & 0.2713724 & 0 & -0.0800528 & 0.452416 & 0.192194 & -0.068245 \\
& 0.8 & 0.2708067 & 0 & -0.0826660 & 0.454631 & 0.198417 & -0.076523 \\
& 0.9 & 0.2701495 & 0 & -0.0853752 & 0.457490 & 0.205298 & -0.085483 \\
& 1.0 & 0.2693890 & 0 & -0.0881727 & 0.461180 & 0.212903 & -0.095180 \\
\hline \hline
\end{tabular}

TABLE V. Critical exponents of the 3D models for RS FP's

\begin{tabular}{|c|c|c|c|c|c|c|c|}
\hline Model & $\mathrm{FP}$ & & $\eta$ & $\nu$ & $\gamma$ & $\beta$ & $\alpha$ \\
\hline \multirow[t]{4}{*}{$\overline{\text { Ising }}$} & $\overline{\mathrm{RS} 1}$ & this work & 0.028 & 0.631 & 1.244 & 0.324 & 0.107 \\
\hline & & Ref. |16 & $0.031(4)$ & $0.630(2)$ & $1.241(2)$ & $0.325(2)$ & $0.110(5)$ \\
\hline & RS2 & this work & 0.028 & 0.672 & 1.329 & 0.345 & -0.015 \\
\hline & & Ref. 17 & $0.030(3)$ & $0.678(10)$ & $1.330(17)$ & $0.349(5)$ & $-0.034(30)$ \\
\hline \multirow[t]{2}{*}{$\overline{\mathrm{XY}}$} & RS1 & this work & 0.029 & 0.667 & 1.318 & 0.343 & -0.001 \\
\hline & & Ref. $\mid 16 \rrbracket$ & $0.034(3)$ & $0.669(1)$ & $1.316(1)$ & $0.346(1)$ & $-0.007(6)$ \\
\hline Heisenberg & $\overline{\mathrm{RS} 1}$ & this work & 0.028 & 0.697 & $\begin{array}{l}1.379 \\
1.2871)\end{array}$ & 0.369 & -0.092 \\
\hline
\end{tabular}

